\title{
An Efficient Parallel Termination Detection Algorithm
}

\author{
A. H. Baker* \\ S. Crivelli ${ }^{\dagger}$ \\ E. R. Jessup $\ddagger$
}

\section{January 6, 2008}

\begin{abstract}
In this paper, we present a new, easy to implement algorithm for detecting the termination of a parallel asynchronous computation on distributedmemory MIMD computers. We demonstrate that it operates concurrently with the main computation, adding minimal overhead, and we prove that it correctly detects termination when it occurs. Experimental results confirm that the termination detection routine imposes an overhead smaller than the experimental uncertainty.
\end{abstract}

Key Words: Termination detection; asynchronous termination; distributed computing

Computing Reviews Category: D.1.3

${ }^{*}$ Center for Applied Scientific Computing, Lawrence Livermore National Laboratory, Box 808 L-551, Livermore, CA 94551 (baker59@llnl.gov). The work of this author was supported by the National Science Foundation under grant no. ACR-93-57812 while in residence at the University of Colorado. Portions of this work were performed under the auspices of the U.S. Department of Energy by University of California Lawrence Livermore National Laboratory under contract No. W-7405-Eng-48.

${ }^{\dagger}$ HPCRD, Lawrence Berkeley Lab, 1 Cyclotron Rd, Mailstop 50F, Berkeley, CA 94720 (crivelli@global.lbl.gov). The work of this author was supported by the National Science Foundation under grant no. ACR-93-57812 and by the Department of Energy under grant no. DE-FG03-97ER25325.

${ }^{\ddagger}$ Department of Computer Science, University of Colorado, Boulder, CO 80309-0430 (jessup@cs.colorado.edu). The work of this author was supported by the National Science Foundation under grant no. ACR-93-57812 and by the Department of Energy under grant no. DE-FG03-97ER25325. 


\section{Introduction}

Information local to any one processor is insufficient to monitor the overall progress of most distributed computations. Typically, a second distributed computation for detecting termination of the main computation is necessary. In order to be a useful computational tool, the termination detection routine must operate concurrently with the main computation, adding minimal overhead, and it must promptly and correctly detect termination when it occurs [13]. In this paper, we present a new algorithm for detecting the termination of a parallel computation on distributed-memory MIMD computers that satisfies all of those criteria.

A variety of termination detection algorithms have been devised. See, for example, $[1,2,5-10,12-14]$. Of these, the algorithm presented by Sinha, Kale, and Ramkumar [13] (henceforth, the SKR algorithm) is unique in its ability to adapt to the load conditions of the system on which it runs, thereby minimizing the impact of termination detection on performance. Because their algorithm also detects termination quickly, we consider it to be the most efficient practical algorithm presently available. The termination detection algorithm presented here was developed for use in the PMESC (Partition Map Embed Solve Communicate) programming library for distributed-memory MIMD computers [3,4].

Like the SKR algorithm, our algorithm adapts to system loads and imposes little overhead. Also like the SKR algorithm, ours is tree-based, and it does not depend on any assumptions about the physical interconnection topology of the processors or the specifics of the distributed computation. In addition, our algorithm requires only half as many tree traverses as the SKR algorithm. We avoid the SKR algorithm's extra tree traversal by augmenting the primary messages, which carry assignments of work in the main computation, with a unique identifier of the latest tree traversal. Finally, our new algorithm is easier 
to implement that the SKR algorithm.

This paper is organized as follows. In section 2, we define our computational model. In section 3, we review the SKR algorithm. We introduce our new algorithm in section 4 , and prove its correctness in section 5 . We discuss its efficiency and present experimental results in section 6 .

\section{Computational Model}

We assume a distributed-memory computer with each processor assigned a different portion of the original problem. Our model of execution is distributed, asynchronous, and dynamic with one process per processor. The processors share computational tasks via message passing.

We distinguish between two concurrent computations: main and termination. The main computation solves the original problem. The termination computation detects the completion of the main computation on all processors. The main computation is further characterized as follows:

- Processors are either active or passive. A processor is active when it is working to complete its assigned computational tasks. Otherwise, it is passive.

- Only active processors can send tasks to other processors.

- Both active and passive processors can receive tasks from other processors.

- An active processor becomes passive only when it finishes its assigned computational tasks.

- A passive processor becomes active only if it receives tasks from another processor.

Messages used by the termination algorithm are called control messages to distinguish them from the primary messages used by the main computation to 
transfer tasks or data. Primary messages may change the status of the receiving processor from passive to active. Control messages cannot change the status of any processor.

The main computation has terminated if and only if

- All processors are passive and

- There are no primary messages left in transit.

A termination detection algorithm must detect both termination conditions.

\section{The SKR Algorithm}

The SKR algorithm [13] is a wave-type algorithm that requires the use of a virtual spanning tree for termination messages, but its description is not dependent on the properties of the physical underlying network topology. The algorithm is asymmetric since a root processor is identified. Neither FIFO communication channels nor synchronous communication are required.

The SKR algorithm does not interfere with the main computation. Processors do not handle the control messages until they are passive, and, in a busy system, few control messages are generated. As a result, the SKR algorithm is very efficient for the computational model described in the previous section. Further discussion of efficiency follows in section 6 .

The SKR algorithm detects termination by counting the number of primary messages sent and received. It is organized in two phases and uses three types of termination messages (initialization, idle, and activity). Communication follows the links of a virtual spanning tree. In the first phase of the algorithm, when a leaf processor becomes passive, it sends an idle message to its parent in the tree containing the numbers of primary messages it has sent and received at that time. Once an internal processor (one that is not a leaf or root) becomes passive, it waits for idle messages (messages signalling that processors are idle) 
from all of its children, adds the received counts to its own count, and forwards the result as an idle message to its parent.

The total counts in any processor represent the number of primary messages sent and received so far by all processors in the subtree rooted at that processor. Thus, if the total counts received by the root of the spanning tree are not equal, termination cannot have occurred, and the root broadcasts a signal to the leaves to reinitiate the first phase. However, if the root's totals are equal, termination may have occurred. Thus, the root broadcasts a signal to the leaves to initiate the second phase. The second phase detects whether any messages are still in transit even though the totals at the root match.

In the second phase, the processors, beginning at the leaves, send activity messages containing their updated counts of primary messages sent and received up the tree. Activity messages are combined in the same way as idle messages are in the first phase. When the root has received activity messages from all of its children, it compares the old totals of messages sent and received with the new ones. If these values are the same, there has been no activity in the system. In this case, the root reports termination. Otherwise, it restarts phase one with a broadcast to the leaves.

Because the progress of this termination detection algorithm is controlled by the root, it requires the broadcasting of a message from the root to the other processors to initiate each of the phases. The SKR algorithm therefore takes four traverses of the spanning tree to detect termination. In the next section, we present a new termination detection algorithm that is in the same class as the SKR algorithm. The new algorithm, however, takes at most two traverses of the spanning tree to detect termination after it occurs. 


\section{The New Algorithm}

The new termination detection algorithm runs on each of the processors in the distributed-memory computer. All communication in the termination procedure takes place up and down a virtual spanning tree of processors. Pseudo-code for the termination algorithm is given in Figure 1.

The algorithm requires one phase and only two types of control messages: down and up. Down messages traverse the tree downwards carrying only the number of the termination sweep (called a sweep_\#) they belong to. Up messages traverse the tree upwards and prompt each processor to evaluate the number of primary messages it has sent minus the number of primary messages it has received. Up messages carry a sweep_\# as well as an accumulated count of sends minus receives from processors below it in the tree.

The downward traversal starts at the root. When the root processor becomes passive, it sends a down message to its children. The sweep_\# included in the down message is assigned by the root. The first time the root initiates a downward traversal it assigns the down messages sweep_\# $=1$. The root increments the sweep_\# by one for each subsequent downward traversal that it initiates so that each termination sweep has a unique sweep_\#. When any processor other than the root becomes passive, it checks to see whether it has received a down message from its parent. If so and if the processor is not a leaf, it forwards the sweep_\# just received to its children as a down message. If the processor is a leaf, it evaluates the difference between the number of primary messages it has sent and received until that point in time. The leaf processor then sends its message count and the sweep_\# contained in the down message as an up message to its parent in the tree. In this way, the leaf processors initiate the upward traversal.

In addition to being attached to the control messages, a termination sweep 
local count of primary messages sent

local count of primary messages received

local count of sends minus receives

$\mathrm{T}$

accumulated count of sends minus receives from children

ccum_count number of children that have sent termination messages

children total number of children that belong to a processor

all_children

sweep_\#

number of the termination sweep

last_msg_sweep_\#

greatest sweep_\# attached to work received by a processor

Downward traversal

if (not root)

check for down message containing a sweep_\# from parent;

if (not leaf)

send down message containing sweep_\# to children;

else $\backslash *$ processor is a leaf $* \backslash$

initiate Upward traversal:

$\mathrm{T}=\mathrm{S}-\mathrm{R}$

send up message containing $\mathrm{T}$ and sweep_\# (from down message) to parent; return to main computation;

\section{Upward traversal}

if (not leaf)

check for up messages from children containing $\mathrm{T}$ and sweep_\#;

if (message received)

accum_count $=\mathrm{T}$ (received) + accum_count;

children ++ ;

if $($ children $==$ all_children $)$

if (last_msg_sweep_\# > local sweep_\#)

$$
\mathrm{T}=\text { INFINITY; }
$$

else

$$
\mathrm{T}=\mathrm{S}-\mathrm{R}
$$

accum_count $=\mathrm{T}+$ accum_count;

if (not root)

send up message containing accum_count and sweep_\# to parent;

set local sweep_\# = sweep_\# in up message just sent;

else $\backslash *$ root processor ${ }^{*} \backslash$

if (accum_count $=0$ )

broadcast termination;

else

go back to Downward traversal;

return to main computation

Figure 1: Pseudo-code for the termination detection algorithm. 
number is attached to all primary messages. Each processor keeps a local quantity called sweep_\#, which is initialized to zero. A processor attaches its local sweep_\# value to all primary messages it sends. A second quantity kept by each processor is last_msg_sweep_\# (also initialized to zero). Each time a processor receives a primary message from another processor, it checks to see if the sweep_\# included with the primary message is greater that its last_msg_sweep_\#. If so, the processor updates its last_msg_sweep_\# to equal the sweep_\# included in the primary message. In this way, the local last_msg_sweep_\# kept by each processor reflects the greatest sweep_\# value attached to any primary message it has received so far.

Although every processor must be passive at some point during the downward traversal, completion of the downward traversal does not signal termination of the main computation: a processor may still receive a primary message and resume work after sending its down or up messages. The purpose of the upward traversal is to detect any primary messages that were in transit during the downward traversal. Note that the upward traversal can begin at a leaf processor before the downward traversal has reached all processors.

An internal processor collects up messages containing local message counts from all of its children. It forms an accumulated message count equal to the sum of its children's local message counts. When the internal processor has received up messages from all of its children, it evaluates its own message count. It then checks to see if it previously received any primary messages from processors that had completed the current termination sweep (i.e., sent both down and up messages in that sweep). Such a message was received if the checking processor has its last_msg_sweep_\# greater than its sweep_\#, requiring it to reset its message count to INFINITY, where INFINITY $+x \equiv$ INFINITY for arbitrary $x$. The processor then adds its own local message count to the accumulated message count and sends the result up to its parent in the tree. Immediately 
after an internal or leaf processor sends an up message, it updates its local sweep_\# to equal the value of the sweep_\# in the up message just sent. In this way, the local sweep_\# kept by each processor reflects the last termination sweep it completed. Note that an internal processor must be passive before it checks for an up message.

When the root receives up messages from all of its children, it determines whether or not termination has occurred. The root forms an accumulated message count (including its own local message count) in the same manner as the internal processors. If the value of its accumulated count is zero, then all the processors have finished, and the root broadcasts a termination order. Otherwise, termination has not occurred and the main computation continues on all processors until the root becomes passive and reinitiates the termination procedure.

This algorithm requires only two traverses of the tree to detect termination. This number is half that of the SKR algorithm presented in [13] because this algorithm requires only two traversals of the tree to detect termination. We do note, however, that for an application with fully synchronous communication, the second phase (requiring 2 traversals) of the SKR algorithm is not required. In both algorithms, one additional traversal is required for the root to broadcast the termination signal to all of the other processors after termination is detected.

A termination algorithm is fault tolerant if it correctly detects termination despite faults that occur at a processor or in the network during the computation. We note that neither our new algorithm nor the SKR algorithm is fault tolerant. 


\section{Proof of Correctness of the New Algorithm}

In this section, we denote the set of processors in the distributed-memory computer by $\mathcal{P}$. Let $s_{m}$ denote the sending of primary message $m$, and let $r_{m}$ denote the receiving of the message. We identify the following sets: $S_{p_{i}}=$ $\left\{s_{m}\right.$, such that $m$ is a message sent by $\left.p_{i}\right\}, S=\left\{S_{p_{i}}, p_{i} \in \mathcal{P}\right\}, R_{p_{i}}=\left\{r_{m}\right.$, such that $m$ is a message recei $\left\{R_{p_{i}}, p_{i} \in \mathcal{P}\right\}$. Let $\# S$ and $\# R$ be the cardinalities of $S$ and $R$ and $T=$ $\# S-\# R$.

THEOREM 1. The termination detection algorithm does not falsely detect termination.

Proof. The proof is by contradiction. Assume that the termination procedure detects false termination. In that case, the procedure has reached the root of the tree with $T=0$ but termination has not yet occurred. Assume that the termination sweep that found $T=0$ had sweep_\# = b. According to the definition of termination given in section 2 , if termination has not occurred then at least one of the following is true: ( $\exists p_{i} \in \mathcal{P}$ such that $p_{i}$ is active) or ( $\exists$ a message $m$ such that $m$ is in transit).

First assume that $p_{i}$ is active after termination is detected. Then $p_{i}$ changed from passive to active after it was visited during the upward traverse, and its local sweep_\# = b. A passive processor can become active only if it receives a primary message from another processor. So $p_{i}$ necessarily received a primary message $\gamma$ from another active processor $p_{j}$. Without loss of generality, we can assume that $p_{j}$ had not yet been visited by the upward traverse when it sent the message although it may or may not have been visited by the downward traverse. (Any set of processors that are busy below the wavefront of the upward traverse must ultimately have been restarted by a message from a busy processor above the wavefront.) Since $p_{i}$ is now active it could either (a) send no primary messages or (b) send a primary messsage to another processor. In case (a), $\# S \neq \# R$ since the sending of $\gamma$ by $p_{j}$ was counted in the termination sweep but 
the receiving of $\gamma$ was not ( $\gamma$ was received by $p_{i}$ after the up travese). Therefore, $T \neq 0$. In case $(\mathrm{b}), p_{i}$ sends a primary message $\beta$ containing sweep_\# $=\mathrm{b}$ to another processor $p_{k}$. Processor $p_{k}$ must be in either of the following 2 stages of the termination procedure:

1. $p_{k}$ has not been visited by the up traverse.

2. $p_{k}$ has been visited by the up traverse (like $\left.p_{i}\right)$

If item 1 is true, then $p_{k}$ 's sweep_\# $<\mathrm{b}$ since it has not completed the upward traverse for this sweep, but its last_msg_sweep_\# $=\mathrm{b}$ because it received $\beta$. Therefore, $p_{k}$ 's up message contains $T=$ INFINITY, and $T$ cannot be 0 upon completion. If item 2 is true, the same situation exists for $p_{k}$ that was just described for $p_{i}$. Processor $p_{k}$ is now active and can either (a) send no primary messages or (b) send a primary messsage to another processor. In case (a), $T \neq 0$ again because the sending of $\gamma$ was counted, but the receiving of $\gamma$ and the sending and receiving of $\beta$ were not.

In case (b), messages can be sent any number of times to processors that have already completed the sweep, and $T \neq 0$ upon completion of the sweep. In this case, the only way to arrive at $\# S=\# R$ is to have another receive counted by a processor that has not completed the upward traverse. Assume a primary message is sent by $p_{k}$ (or any other active processor that has completed the current sweep) to any processor $p_{e}$ that has not completed the upward traverse. Processor $p_{e}$ sets T=INFINITY when it sends its up message since its last_msg_sweep_\# is be greater than its sweep_\#. Thus, our first assumption is false.

Now assume that there exists at least one message in transit at the completion of the termination sweep. Since $T=0$, then by the definitions of $S$ and $R$ there exist at least two messages in transit, $m_{1}$ and $m_{2}$, such that the sending of $m_{1}$ and the receiving of $m_{2}$ have been counted but the receiving of $m_{1}$ and 
the sending of $m_{2}$ have not. Thus, there are messages $m_{1}$ and $m_{2}$ such that $\left(s_{m_{2}} \notin S\right.$ and $\left.r_{m_{2}} \in R\right)$ and $\left(s_{m_{1}} \in S\right.$ and $\left.r_{m_{1}} \notin R\right)$. Let $p_{k}$ be the processor that sends $m_{2}$ and $p_{j}$ be the processor that receives $m_{2}$. Because $s_{m_{2}}$ is not in $S$ then $m_{2}$ was sent after $p_{k}$ was visited in the upward traverse. Note that $m_{2}$ had to contain sweep_\# $=\mathrm{b}$ since the sending node $p_{k}$ had already been visited by the upward traverse, making $p_{j}$ 's last_msg_sweep_\# $\geq \mathrm{b}$. Since $r_{m_{2}}$ is in $R$ then it occurred before processor $p_{j}$ was visited by the upward traverse. Therefore, when the upward traverse visits processor $p_{j}, p_{j}$ will have last_msg_sweep_\# $\geq \mathrm{b}$ and sweep_\# $<$ b, so T=INFINITY. Thus, our second assumption is also false. $\diamond$

We have proved that when termination is detected the system has terminated. It is also clear that our algorithm will detect termination if it has occurred. By the definition given in section 2, no messages are in transit and all processors are passive when termination has occurred. In that case, the root processor is passive and initiates a downward sweep. Because no messages are in transit during the downward sweep and because no passive processor can become active in the absence of any active processors, the last_msg_sweep_\# cannot be greater than the sweep_\# for any processor during the upward sweep. Therefore, the root will have $T=0$ on completion of the upward sweep, signaling that termination has occurred.

\section{Efficiency of the New Algorithm}

The efficiency of a termination detection algorithm has traditionally been equated with the number of control messages the algorithm creates. In particular, an algorithm is classified as "message optimal" if it uses a number of control messages on the order of the number of primary messages sent by the main computation [8]. We note, however, that the number of control messages does not 
accurately represent the efficiency of the algorithm because control messages are handled only by passive processors. In this case, a better measure of efficiency is the overall impact the termination detection computation has on the performance of the main computation. Thus, an efficient termination detection algorithm must not interfere with the main computation. Furthermore, it must be able to detect both of the termination conditions identified in section 2 and communicate them to all of the processors quickly [13]. The SKR algorithm satisfies both of these efficiency requirements and typically requires far fewer control messages than the message optimality bound would dictate [13]. In this section, we present experimental results to demonstrate that our algorithm is likewise efficient in practice.

We examine a problem contrived to isolate the impact of our termination detection routine in a roughly worst case scenario. The experiments were carried out on the ASCI Blue-Pacific machine (an IBM RS/6000 system) at Livermore National Laboratory. Test programs use Fortran (mpxlf -O), and MPI [11] is used for message passing.

In this problem, all processors begin by completing one short-lived task. When the root completes its task, it sends work to a given leaf processor and becomes passive. When that leaf processor completes its task, it sends work to the root and becomes passive itself. This cycle repeats five times. In the static implementation, the root and one leaf processor terminate computation after the fifth cycle. The remaining processors terminate computation upon completing the preliminary task. Thus, no termination detection routine is actually needed. For purposes of comparison, however, we have written a dynamic implementation that follows the same steps but also employs our termination detection algorithm. The expected impact of termination detection on this problem is high as the runtime is dominated by the communication cycles between root and leaf, and the root processor initiates a new termination detection pro- 


\begin{tabular}{|c|c|c|c|}
\hline $\begin{array}{c}\text { Number of } \\
\text { Processors }\end{array}$ & Dynamic & Static & Ratio \\
\hline 8 & $4.750 \pm 0.012$ & $4.741 \pm 0.010$ & 1.001 \\
\hline 16 & $4.379 \pm 0.008$ & $4.372 \pm 0.008$ & 1.001 \\
\hline 32 & $4.508 \pm 0.009$ & $4.507 \pm 0.018$ & 1.000 \\
\hline 64 & $4.404 \pm 0.010$ & $4.400 \pm 0.009$ & 1.001 \\
\hline 128 & $4.486 \pm 0.007$ & $4.476 \pm 0.014$ & 1.002 \\
\hline 256 & $4.629 \pm 0.007$ & $4.620 \pm 0.010$ & 1.002 \\
\hline 512 & $4.748 \pm 0.087$ & $4.737 \pm 0.066$ & 1.002 \\
\hline
\end{tabular}

Table 1: Total execution times (in seconds) with and without termination detection on ASCII Blue-Pacific. Times reported are averages and standard deviations of times for 250 runs.

cedure every time it changes from active to passive. Nonetheless, the results reported in Table 1 show that adding termination detection has little effect on the total runtime, even in this worse case. The times reported in the table are the averages and standard deviations of the times recorded for 250 runs of the program. As the nonzero standard deviations show, differences in system load, numbers of simultaneous users, and other operational factors cause fluctuations in experimental timings. These ratios of dynamic to static times demonstrate that the termination detection routine imposes an overhead smaller than the experimental uncertainty.

\section{References}

[1] S. Chandrasekaran and S. Venkatesan. A message-optimal algorithm for distributed termination detection. Journal of Parallel and Distributed Computing, 8:245-252, 1990.

[2] M. Chandy and J. Misra. An example of stepwise refinement of distributed programs: Quiescence detection. ACM Trans. on Programming Languages and Systems, 8(3):326-343, 1986. 
[3] S. Crivelli. A programming paradigm and library for distributed-memory computers. PhD thesis, Dept. of Computer Science, University of Colorado at Boulder, 1995.

[4] S. Crivelli and E.R. Jessup. The PMESC programming library for distributed-memory MIMD computers. Journal of Parallel and Distributed Computing, 57:295-321, 1999.

[5] E.W. Dijkstra and C.S. Scholten. Termination detection for diffusing computations. Information Processing Letters, 11(1):1-4, 1980.

[6] M. Filali, P. Mauran, G. Padiou, P. Queinnec, and X. Thirioux. Refinementbased validation of an algorithm for detecting distributed termination. Lecture Notes in Computer Science, 1800:1027-1036, 2000.

[7] N. Mahapatra and S. Dutt. An efficient delay-optimal distributed termination detection algorithm. Technical Report 2001-16, Department of Computer Science and Engineering, University at Buffalo, 2001.

[8] J. Matocha and T. Camp. A taxonomy of distributed termination detection algorithms. The Journal of Systems and Software, 43:207-221, 1998.

[9] F. Mattern. Global quiescence detection based on credit distribution and recovery. Information Processing Letters, 30:195-200, 1989.

[10] F. Mattern. Efficient algorithms for distributed snapshots and global virtual time approximation. Journal of Parallel and Distributed Computing, 18:423$434,1993$.

[11] Message Passing Interface Forum. MPI2: A message passing interface standard. High Performance Computing Applications, 12(1-2):1-299, 1998.

[12] S.P. Rana. A distributed solution of the distributed termination problem. Information Processing Letters, 17:43-46, 1983. 
[13] A. Sinha, L.V. Kale, and B. Ramkumar. A dynamic and adaptive quiescence detection algorithm. Technical Report 93-11, Parallel Programming Laboratory, Department of Computer Science, University of Illinois at Urbana-Champaign, 1993.

[14] G. Tel and F. Mattern. The derivation of distributed termination detection algorithms from garbage collection schemes. ACM Trans. on Programming Languages and Systems, 15(1):1-35, 1993. 